\title{
A pre-post study of behavioural determinants and practice change in Ugandan clinical officers
}

L M T Byrne-Davis, ${ }^{1}$ BSc Hons, MSc, PhD, CPsychol, PFHEA; M J Jackson, ${ }^{2}$ BSc Hons, MB ChB, PGCert; R McCarthy, ${ }^{3}$ RN, RM, MSc, PGCE, SoM, PhD; H Slattery, ${ }^{4} \mathrm{RN}$, BSc, PGCE; G Yuill, ${ }^{2} \mathrm{MB}$ ChB, FRCA; A Stevens, ${ }^{5} \mathrm{RGN}$; G J Byrne, ${ }^{6} \mathrm{MB}$ ChB, MD, FRCS, MAME, NTF; H Parry, ${ }^{1}$ MB ChB;

S Ramsden, ${ }^{7}$ MB ChB, BMedSc, DTM\&H; H Muwonge, ${ }^{8}$ MB ChB, PGD, PPM, MUMA; M Johnston, ${ }^{9}$ BSc, PhD, CPsychol, CClinPsych;

C J Armitage, ${ }^{10}$ BA Hons, PhD, CPsychol, AFBPS; S Cook, ${ }^{5}$ RN; S Whiting, ${ }^{11}$ RN, ENB100; J Gray, ${ }^{12}$ RN; J Hart, ${ }^{1}$ BSc Hons, MSc, PhD, CPsychol, AFBPS PFHEA

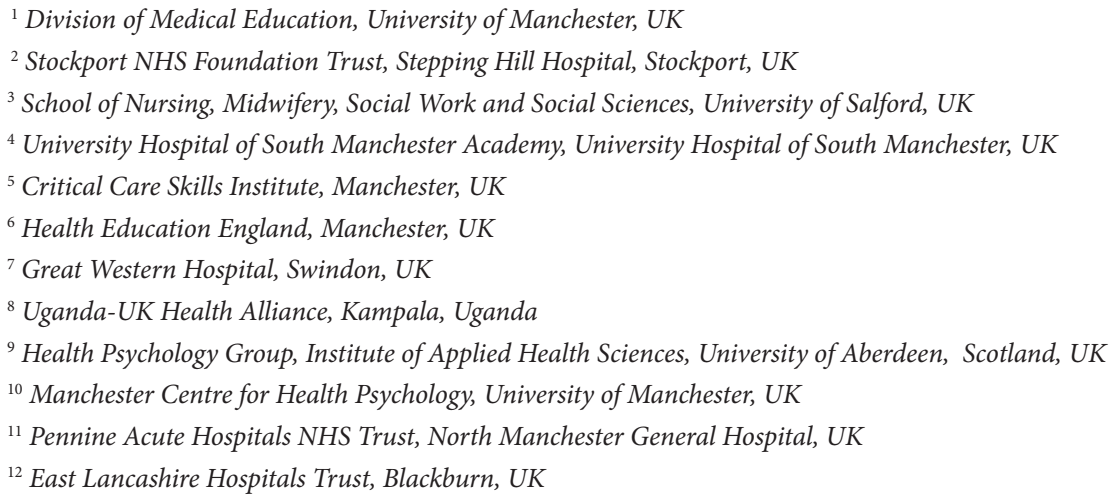

Corresponding author: L M T Byrne-Davis (lucie.byrne-davis@manchester.ac.uk)

\begin{abstract}
Background. Understanding the drivers of 'provider behaviour' has been highlighted as one of the six domains of behaviour change in strengthening healthcare systems.

Objectives. To assess changes in healthcare provider behaviour, i.e. use of the Airway, Breathing, Circulation, Disability, Exposure (ABCDE) approach in acute illness management, after participating in a 1-day course on the assessment and management of acutely ill patients. We aimed to assess whether changes in psychological determinants of the $\mathrm{ABCDE}$ approach were associated with changes in the use of the approach.

Methods. We used a pre-post design to study self-reported change in behaviour after a 1-day training course from pre-course to follow-up 1 month later. We also measured psychological determinants of behaviour immediately before and after and at 1-month follow-up. We explored if changes in psychological determinants were associated with change in practice 1 month later.

Results. We found the following: firstly, use of the ABCDE approach increased at 1 month post-course from a median use of $50-90 \%$. Secondly, the increase in the $\mathrm{ABCDE}$ approach was associated with a positive change in only one of the determinants of practice from pre- to post-course: perception of environmental determinants $(r=0.323 ; p<0.05)$. Finally, there were no other significant associations with practice change or practice at follow-up. Conclusions. Change in perceptions of availability of resources was associated with increased use of an ABCDE approach, but evidence was limited owing to the pre-post design.
\end{abstract}

Afr J Health Professions Educ 2018;10(4):220-227. DOI:10.7196/AJHPE.2018.v10i4.994

The importance of behavioural science in advancing health in low- and middle-income countries (LMICs) was highlighted in a journal funded by the US Agency for International Development (Global Health Science and Practice).$^{[1]}$ In particular, the authors outlined six domains of behaviour change that were building blocks of global health. One of these domains was 'provider behaviour' and included understanding healthcare workers' capabilities, opportunities and motivations to provide high-quality care. We propose that understanding provider behaviour is most urgent in the areas of healthcare that have been shown to impact greatly on patient mortality and morbidity, e.g. management of the critically ill patient.

Management of the deteriorating and critically ill patient is a key activity in acute healthcare facilities. Firth and Ttendo ${ }^{[2]}$ emphasised the need for recognition, assessment and management of the critically ill in Uganda and other low-income countries. This need is great because many patients present to hospital in a critical state owing to certain factors, including underlying health issues (e.g. malnutrition); present to hospital at a late stage owing to the time taken to travel to hospital; and seek help at a late stage of an illness because of the need to pay fees. A systematic review of critical care in LMICs found that many health professional students had limited training in the assessment and management of acutely ill patients. ${ }^{[3]}$ It is clear, therefore, that there is a need to educate and train staff in the management of acute illness in LMICs.

There are many courses that teach the recognition and management of acutely ill people. Examples are the 1-day Acute Illness Management (AIM) 
and its sister course Maternal Acute Illness Management (M-AIM), which teach an Airways, Breathing, Circulation, Disability, Exposure (ABCDE) approach to acute illness recognition and management. AIM and M-AIM have previously been shown to improve the knowledge of participants immediately after the course. ${ }^{[4,5]}$ Further research on these two courses revealed barriers and facilitators to healthcare professionals using their new knowledge and skills in practice. ${ }^{[6]}$ In response to these barriers, the Greater Manchester Critical Care Skills Institute developed AIM4Africa, a 1-day interactive course, which could increase the implementation of knowledge and skills in practice by addressing barriers and focusing on facilitators.

Research investigating how healthcare professionals put new knowledge and skills into practice, explores variables, i.e. 'behavioural determinants'. These variables typically influence whether someone executes a particular behaviour and can be categorised as capability, opportunity and motivation..$^{[7]}$ There are many behavioural determinants in each category. Eccles et al. ${ }^{[8]}$ described behavioural determinants that had been associated specifically with changes in provider behaviour. These include both deliberative/reflective processes and automatic or habitual processes, ${ }^{\left[{ }^{[9]}\right.}$ and were constructs in: (i) theory of planned behaviour; (ii) social cognitive theory; (iii) selfregulation model; (iv) learning theory; $(v)$ implementation intentions; (vi) knowledge, attitudes, behaviour; and (vii) stages of change, as specified by the precaution adoption process. Innovations in practice are frequently investigated using the Promoting Action on Research Implementation in Health Services (PARIHS) framework. ${ }^{[10]}$ The constructs in PARIHS are: (i) impact of having facilitation/support while attempting to change practice; and (ii) perception of the strength of evidence change in practice. Moreover, burnout has frequently been shown to be high in healthcare professionals. ${ }^{[11,12]}$ It is not known whether high levels of burnout might negatively impact practice change.

Many training courses assess whether programmes are effective by measuring behavioural determinants within the category of 'capability', e.g. knowledge and skills. However, it is rare for training courses to assess whether programmes are effective by examining changes in behavioural determinants that fall into the categories of opportunity and motivation. Therefore, there is little understanding of whether a training course that encourages an ABCDE approach to acute illness management changes behavioural determinants in the categories of capability, opportunity and motivation. There is also little understanding about whether changes in any of the behavioural determinants are associated with changes in use of the $\mathrm{ABCDE}$ approach. To address these gaps, our study sought to examine the following:

- Determine if attendance at an acute illness management course tailored to the low-resource setting (AIM4Africa) would be associated with change of specific behaviour, specifically using an $\mathrm{ABCDE}$ approach to care for acutely ill patients from before the course to 1 month later. This was done by measuring and comparing self-report use of an $\mathrm{ABCDE}$ approach immediately before the course and 1 month later.

- Explore if AIM4Africa changed any behavioural determinants regarding the use of an $\mathrm{ABCDE}$ approach from before to immediately after the course by measuring these.

- Explore associations between changes in behavioural determinants and in the use of an $\mathrm{ABCDE}$ approach by investigating data statistically for associations.

- Explore associations between behavioural determinants that occur at the point of putting new knowledge and skills into practice (i.e. implementation support, feedback and burnout) and behaviour by exploring the data statistically for association.

\section{Methods}

In a pre-post design, we examined self-reported use of an $\mathrm{ABCDE}$ approach by clinical officers in Gulu, Uganda, prior to the AIM4Africa course and 1 month later. We measured behavioural determinants before and immediately after the course and at 1 month follow-up. We compared changes in behavioural determinants from pre- to post-course with changes in the use of an $\mathrm{ABCDE}$ approach pre-course to 1 month follow-up.

\section{Participants}

Clinical officer students ( $N=77)$ in year 3 (final year, 2014 - 2015) of their course enrolled in AIM4Africa. These Ugandan students train to become licensed, fully qualified medical practitioners. Completion rates were 73 of 77 (95\%) for pre-course and 70 of 77 (91\%) for follow-up (i.e. $93 \%$ of the pre-course completers). The participants' mean age was 24 (standard deviation 2.3; range 21 - 35) years and $71 \%$ were men.

\section{Measures}

\section{Applied knowledge}

We assessed applied knowledge by 'single best answer' multiple-choice questions (MCQs) with between 4 and 6 response options, authored by the AIM steering group. Items were divided into two papers and each participant had the same paper pre- and post-course. Participants scored 1 point for each correct answer and a percentage score was calculated for overall knowledge.

\section{Capability checklist (self-report)}

Measured with a 49-item checklist from the core capabilities in the AIM4Africa course, participants were asked to indicate if they could perform each task or skill independently ( 2 points), with some support (1 point), not at all (0 points) or do not know (missing data). Scores ranged from 0 to 98.

\section{Behavioural determinants' questionnaire and usual behaviour}

There were 18 behavioural determinants and 46 questions. We assessed usual behaviour by: (i) two questions requiring strength of agreement, with statements about how typical it was for the participant to conduct that behaviour in that context; and (ii) estimates of the number of patients seen in the past month, who they thought might have been acutely unwell and estimates of with how many of these patients they used the ABCDE approach. We based the style of questions on the Research-Based Education and Quality Improvement (REBEQI) Manual, ${ }^{[15]}$ which outlines methods for creating questions assessing the construct in the theory of planned behaviour. Names, numbers of questions and minimum and maximum possible scores of each construct are given in Table 1.

\section{Procedure}

\section{Before the AIM4Africa course: Pre-course}

The questionnaires were integrated into the course timetable; information emphasised that the evaluation was not a prerequisite to take the course; all learners consented to take part; and participants completed the pre-course measures. 
Table 1. Constructs, descriptions, number of items and range of possible scores for behavioural determinants and usual behaviour

\begin{tabular}{|c|c|c|}
\hline Construct & Description & $n$ (range) \\
\hline $\begin{array}{l}\text { Awareness of need for } \\
\text { change }\end{array}$ & $\begin{array}{l}\text { Being aware that a problem exists and thinking about overcoming it, but without having made a plan or } \\
\text { a commitment to take action }\end{array}$ & $1(1-7)$ \\
\hline Behavioural expectation & One's expectation that one will perform a behaviour & $1(0-10)$ \\
\hline Habit & A behaviour or pattern of behaviours that tends to occur unconsciously in response to a situation or context & $2(1-7)$ \\
\hline Burnout & $\begin{array}{l}\text { A prolonged response to chronic emotional and interpersonal stressors at work and defined by three dimensions } \\
\text { of exhaustion, cynicism and inefficacy }\end{array}$ & $3(1-7)$ \\
\hline Implementation support & Having specific individuals who facilitate the carrying out of a behaviour & $3(1-7)$ \\
\hline Action control & $\begin{array}{l}\text { Self-regulatory processes; processes that involve initiating, inhibiting, modulating or monitoring } \\
\text { a particular behaviour }\end{array}$ & $3(1-7)$ \\
\hline General intention & Motivation or decision to act in a particular goal-directed way & $3(1-7)$ \\
\hline Attitudes to behaviour & Positive and negative beliefs and feelings towards a behaviour & $5(1-7)$ \\
\hline Outcome expectancies & Perceived likelihood that one or more outcomes will occur having acted in a particular way & $3(1-7)$ \\
\hline Subjective norms & $\begin{array}{l}\text { Rules and standards that are understood by members of a group, that guide and/or constrain social behaviour } \\
\text { without the influence of laws }\end{array}$ & $3(1-7)$ \\
\hline Self-efficacy & Self-efficacy is the extent or strength of one's belief in one's ability to complete tasks and reach goals & $1(1-7)$ \\
\hline $\begin{array}{l}\text { Perceived behavioural } \\
\text { control }\end{array}$ & An individual's perceived ease or difficulty of performing a behaviour & $2(1-7)$ \\
\hline Knows how & Knowing how to perform a behaviour or achieve a goal & $2(1-7)$ \\
\hline Strength of evidence & $\begin{array}{l}\text { Codified and non-codified sources of knowledge, including research evidence, clinical experience, } \\
\text { e.g. professional craft knowledge, patient preferences and experiences and local information, in favour of } \\
\text { performing a new behaviour }\end{array}$ & $1(1-7)$ \\
\hline Action planning & A process that links goal-directed responses to situational cues specifying how, where and when to act & $3(1-7)$ \\
\hline Coping planning & $\begin{array}{l}\text { Making a plan of what to do to achieve one's goals when there are difficulties in executing one's initial } \\
\text { action plan }\end{array}$ & $4(1-5)$ \\
\hline Feedback & Response of other people after performing a particular behaviour & $3(1-7)$ \\
\hline $\begin{array}{l}\text { Environmental } \\
\text { determinants }\end{array}$ & Influence of the physical environment and surroundings on behaviour & $3(1-7)$ \\
\hline Self-report behaviour & $\begin{array}{l}\text { Percentage of possible times of behaviour }=(\text { number of times you performed the behaviour/number of times } \\
\text { appropriate for performance of the behaviour) multiplied by } 100\end{array}$ & $2(0-100)$ \\
\hline Usual behaviour & To what extent one performs a behaviour in a particular situation or context & $2(1-7)$ \\
\hline
\end{tabular}

\section{After the AIM4Africa course: Post-course and follow-up}

On completion of the course, participants completed the pre-course questionnaires again, without questions about usual behaviour. One month later, the researchers attended the 4 local health centres, where participants were placed for 3 days (20 - 22 students at each facility). Questionnaires were administered during breaks in clinical practice.

\section{Analyses}

\section{Change in use of the $\mathrm{ABCDE}$ approach}

We compared median behaviour pre-course and at follow-up using the Wilcoxon signed-rank test. We calculated a change score for behaviour by subtracting the percentage of acutely ill people for whom participants reported taking an $\mathrm{ABCDE}$ approach pre-course from the percentage at follow-up.

Use of the ABCDE approach is advocated for all people suspected to be acutely ill. Therefore, we dichotomised the self-reports of behaviour into those using the ABCDE approach in $100 \%$ of people they suspected were about to become acutely ill and those not using the approach $100 \%$ of the time. We reported frequencies and percentages of participants always using the $A B C D E$ apparoach and those not using $A B C D E$ continuously precourse and at 1 month follow-up. These were compared using the $\chi^{2}$ test to see if frequency of maximal use of the $\mathrm{ABCDE}$ approach had changed.
Change in behavioural determinants from pre- to immediately post-course We calculated a change score for each behavioural determinant by subtracting pre-course scores for each construct from immediately post-course scores. We also compared median behavioural determinant scores pre-course and immediately post-course using the Wilcoxon signed-rank test.

Since the scale can be split into agree or disagree (with neutral included in the disagree category, as it is not 'agreeing'), we also calculated the number and percentage of participants who agreed (i.e. were not neutral or disagreed) with the statements at each time point by calculating an agree/ not agree variable for each behavioural determinant, $\geq 4.5$ being agree and $1-4.4999$ being not agree. We used the $\chi^{2}$ test to determine if the number of those agreeing changed from pre-course to post-course.

\section{Internal consistency reliability of behavioural determinant scales}

For the constructs with $>1$ item assessing the construct, Cronbach's alpha analyses were conducted to assess internal consistency reliability. Where these showed poor internal consistency $(<0.2)$, we reported the results but did not use the construct in any further inferential analyses.

\section{Distribution of variables}

We conducted the Shapiro-Wilk test of normality on each variable to determine whether to use parametric or non-parametric inferential 
statistics. Of the 36 variables measured at pre- and post-course and followup, 5 were normally distributed. The others were Shapiro-Wilk $0.184-$ $0.969, \mathrm{df}=29$ and $p<0.05$. We therefore continued with presenting median and interquartile ranges as descriptions of the variables and conducted non-parametric inferential statistics.

\section{Change scores}

We tested change score variables for normality to determine whether to use parametric or non-parametric inferential statistics, again using ShapiroWilk. Of the 11 change scores calculated, 3 were normally distributed. The test of normality for the others were Shapiro-Wilk $0.605-0.983, \mathrm{df}=54$ and $p<0.05$. We therefore continued with presenting median and interquartile ranges as descriptions of the variables and conducted non-parametric inferential statistics.

Change in behavioural determinants associated with behaviour change Using Spearman's rank test, we correlated all change in behavioural determinants (from pre- to immediately post-course) with change in behaviour (from pre-course to follow-up) to establish if any behavioural determinant changes during the course were associated with behaviour change from pre-course to follow-up.

\section{Missing data}

For constructs with multiple items, where there was one missing data point, medians or means were calculated using the remaining items. Where there was more than one missing data point, data from the whole construct were excluded for that participant.

\section{Ethical approval}

This project had approval from the Lacor Institutional Review Board and Ministry of Health in Uganda. Confirmation was given from the University of Manchester Research Ethics Committee in the UK that they did not require additional formal approval, as the work would be considered evaluation of training.

\section{Results}

\section{Change in use of the $A B C D E$ approach (study aim 1)}

The median use of the ABCDE approach was 50\% at pre-course and $90 \%$ at follow-up; a Wilcoxon signed-rank test indicated that this was a statistically significant change $(z-3.941 ; p<0.001)$.

At pre-course, 16 of 66 (24\%) participants reported using the $\mathrm{ABCDE}$ approach in $100 \%$ of acutely ill patients. At follow-up, it was 34 of 71 (45\%). A $\chi^{2}$ test indicated that this increase was not statistically significant (Table 2).

\section{Behavioural determinants} Internal consistency reliability

We assessed internal consistency reliability at the first time of measurement, e.g. pre-course. Subjective norms and perceived behavioural control were not considered to be measured reliably enough to be included in further analyses (Table 3).

\section{Stage of change}

Before the course, almost half of participants reported that they had already started using an $\mathrm{ABCDE}$ approach, although this number decreased to only a fifth after the course. Those reporting that they had made a decision to use an $\mathrm{ABCDE}$ approach rose from $23 \%$ to $62 \%$ from pre- to post-course. This finding perhaps indicates that participants thought that they were using an $\mathrm{ABCDE}$ approach before the course; yet, after attending the course, they realised they were not and then made the decision to try this method (Table 4).

\section{Change in behavioural determinants (study aim 2)}

Pre-course medians tended towards the top of the range for each scale. Measures with lower scores were action control (median 4.7) and action planning (median 5.0). Burnout had a median of 3.7, but, in this case, a lower score is positive, as it indicates a lower level of burnout.

The Wilcoxon signed-rank test showed that there were significant increases in scores for capability, knowledge (measured by MCQ), general intention, attitudes to behaviour, outcome expectancies and self-efficacy, knows how, strength of the evidence and action planning after the course, with fewer participants with lower scores on these measures (Table 3). Most of the questions were answered by most of the participants. However, the question about behavioural expectation was answered by 40 of $73(55 \%)$ participants at pre-course, 54 of $77(70 \%)$ at post-course and 68 of $71(96 \%)$ at follow-up.

\section{Change in behavioural determinants associated with change in use of the ABCDE approach (study aim 3)}

Change in use of an ABCDE approach from pre-course to follow-up was statistically significantly correlated with change in perception of environmental determinants from pre- to post-course $(r=0.323 ; p<0.05)$. No other change in behavioural determinants from pre- to post-course was statistically significantly correlated with change in behaviour from precourse to follow-up.

\section{Association between behaviour (at follow-up) and implemen-} tation support, feedback and burnout (study aim 4)

Spearman's rank correlations illustrated that there were no statistically significant correlations between implementation support, feedback, burnout and behaviour at follow-up.

\section{Discussion}

Participation by Ugandan clinical officers in an acute illness management programme was statistically significantly associated with an increase in self-reported use of an $\mathrm{ABCDE}$ approach to treat acutely ill patients from pre-course to follow-up 1 month after course completion. Multiple behavioural determinants, which were measured at pre- and post-course, changed. The only changes from pre- to post-course that were statistically significantly associated with a change in use of the $\mathrm{ABCDE}$ approach from pre-course to follow-up were environmental determinants, e.g. perception of time and resources. This is significant, because there are many theoretical determinants of change, ${ }^{[8]}$ but this study indicates that, in a low-resource environment, the perception of environmental determinants is crucial. It is important to distinguish actual environmental determinants, e.g. having time and resources, from what we assessed in our study, i.e. perceptions of time and resources. It is clear that these were perceptions, as they changed from pre- to post-course when actual time and resource had not altered. The setting is known to be a low-resource one; therefore, it is possible that 


\section{Research}

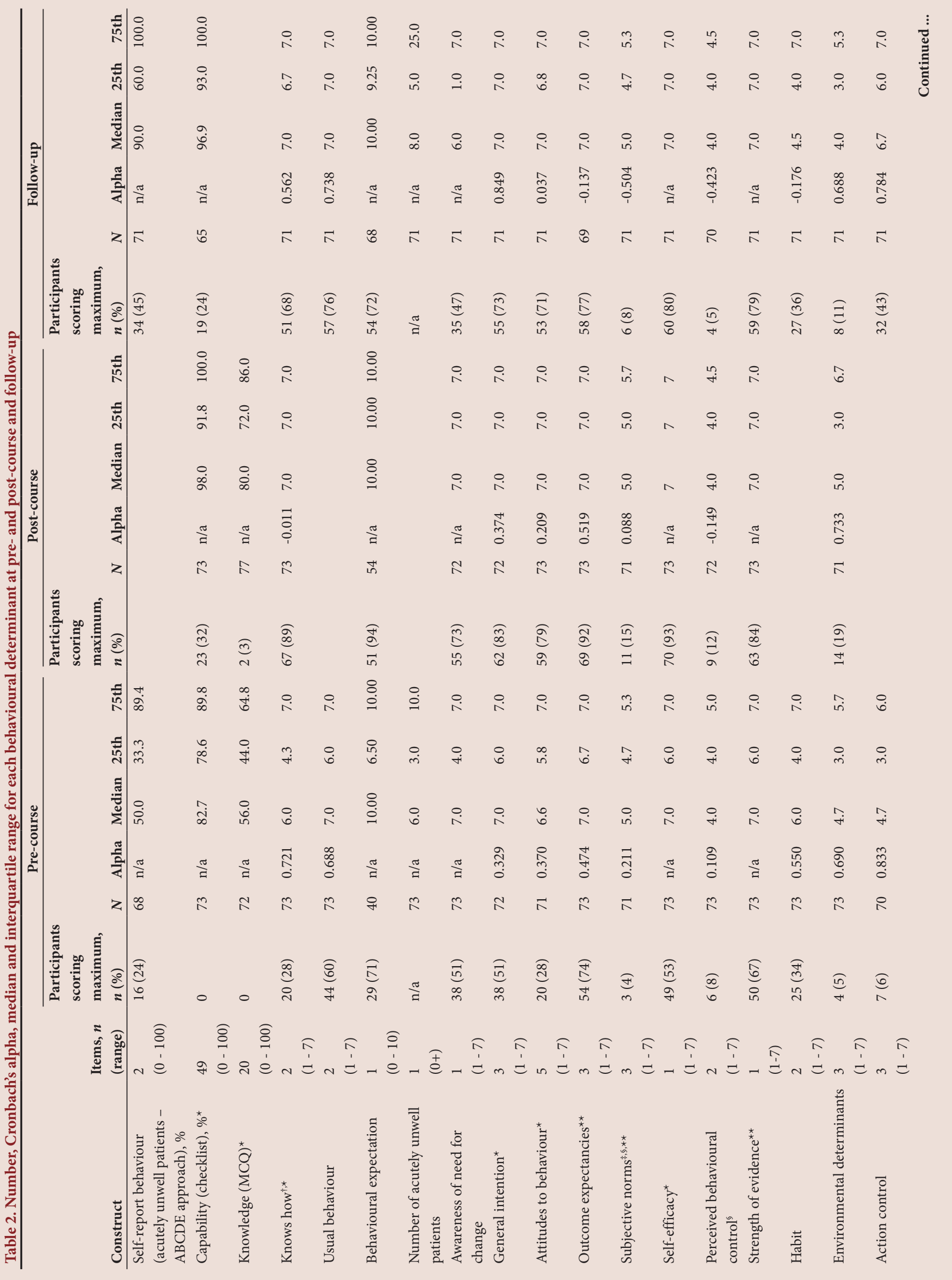


people would tend to report lack of resources, time and equipment. This changed over the course of a day, with no change in the actual resources. This could be a methodological limitation in using the method of selfreporting; yet, the internal consistency reliability of the three questions about these environmental determinants changed from moderate to high, at 0.690 . In terms of the direction of change, there were changes in both directions. Some people reported less availability of resources, while some reported higher availability. AIM4Africa was specifically modified for low-resource settings and one modification is offering alternatives to goldstandard management techniques, such as high concentrations of oxygen. In terms of behaviour change, techniques offering these alternatives are likely to help the participants form coping plans. A coping plan is one that an individual creates to cope if something happens that makes what they intend to do more difficult. ${ }^{[13,14]}$ In the example of a lack of the goldstandard concentration of oxygen, offering the participant the option of using the highest concentration available, will encourage them to formulate a plan to use the highest concentration available should the gold-standard concentration not be available. The observed change in the participants' views of the availability of time and resources might, therefore, be related to these features of the course. A positive change in the perceptions of availability of time and resources from the start to the end of the course was associated with an increase in target behaviour from pre-course to followup. Therefore, in this case, perceptions of a resource can change, which might be associated with a change in behaviour 1 month later. Based on this finding, we recommend that future research examine whether a change in perception of environmental determinants is brought about by other courses, and whether this is related to a change in other types of practice, particularly coping planning behaviour change techniques.

It is significant that changes in pre- to post-behavioural determinants in the category of capability were not associated with change in use of the $\mathrm{ABCDE}$ approach. This finding is important because, as stated above, it is typical for training courses to assess changes in capability (e.g. knowledge) and not to assess changes in the categories of opportunity and motivation. If our finding that perception of opportunity is a key driver of change in practice is replicated in other courses, it would indicate that courses should broaden the way in which they assess course outcomes.

We found that it was feasible to create a pre- and post-course evaluation that assessed multiple behavioural determinants and self-reported behaviour. Exploration of the behavioural determinants of practice and their associations with both the techniques used in the courses and the subsequent adoption of new practices would enhance educational practice. An understanding of the 'active ingredients' of training courses, how they change the psychology of the trainees and how these relate to change in provider behaviour, would provide further insights into the design of new education tools that aim to change practice and ways of understanding if the tools are effective.

\section{Study limitations}

The behavioural measure was self-reported. Self-reported measures are useful where observation is not possible but are potentially influenced by forgetting and social desirability. Verification of self-reported changes in practice through observation would add to the strength of the evidence. We conducted a pre-post study; therefore, we cannot conclude that the content of the course was responsible for the change in practice. 
Table 3. Stage of change of participants at pre- and post-course and follow-up

\begin{tabular}{|c|c|c|c|}
\hline Stage of change & $\begin{array}{l}\text { Pre-course, } \\
n(\%)\end{array}$ & $\begin{array}{l}\text { Post-course, } \\
n(\%)\end{array}$ & $\begin{array}{l}\text { Follow-up, } \\
n(\%)\end{array}$ \\
\hline I have not yet thought about using an $\mathrm{ABCDE}$ approach with patients who might be acutely unwell & $4(5)$ & $1(1)$ & $1(1)$ \\
\hline $\begin{array}{l}\text { It has been a while since I have thought about using an } \mathrm{ABCDE} \text { approach with patients who might be } \\
\text { acutely unwell }\end{array}$ & $8(11)$ & $1(1)$ & 0 \\
\hline $\begin{array}{l}\text { I have thought about it and decided that I will not use an ABCDE approach with patients who might be } \\
\text { acutely unwell }\end{array}$ & 0 & 0 & 0 \\
\hline I have decided that I will use an $\mathrm{ABCDE}$ approach with patients who might be acutely unwell & $17(23)$ & $46(62)$ & $20(27)$ \\
\hline I have already started using an $\mathrm{ABCDE}$ approach with patients who might be acutely unwell & $36(49)$ & $15(20)$ & $46(62)$ \\
\hline $\begin{array}{l}\text { I have already done something about managing patients who might be acutely unwell, not using } \\
\text { an } A B C D E \text { approach }\end{array}$ & $1(1)$ & 0 & $2(3)$ \\
\hline Not completed (missing) & $8(11)$ & $11(15)$ & $5(7)$ \\
\hline
\end{tabular}

Table 4. Medians, interquartile ranges of pre- to post-course change scores for behavioural determinants and pre- to follow-up change scores for use of $\mathrm{ABCDE}$ approach and habit

\begin{tabular}{lll}
\hline Change variable & $n$ & Median (IQR) \\
\hline Behaviour change pre-course to follow-up & 64 & $18.3(-2.1-50.0)$ \\
Capability checklist change pre- to post-course & 66 & $11.2(6.1-17.6)$ \\
Awareness of need for change pre- to post-course & 72 & $0.0(0.0-1.0)$ \\
Behavioural attitudes pre- to post-course & 71 & $0.4(0.0-0.8)$ \\
Outcome expectancies pre- to post-course & 73 & $0.0(0.0-0.2)$ \\
Self-efficacy pre- to post-course & 73 & $0.0(0.0-1.0)$ \\
Strength of the evidence pre- to post-course & 73 & $0.0(0.0-0.5)$ \\
Habit pre-course to follow-up & 69 & $0.0(-1.5-1.0)$ \\
Environmental determinants pre- to post-course & 69 & $0.0(-1.7-0.8)$ \\
Action planning pre- to post-course & 69 & $1.7(1.0-3.0)$ \\
Action control pre- to post-course & 66 & $2.0(0.3-3.7)$ \\
ABCDE = Airway, Breathing, Circulation, Disability, Exposure; IQR = interquartile range. & &
\end{tabular}

We also aimed to explore if any behavioural determinants of the use of an $\mathrm{ABCDE}$ approach changed from before to immediately after the course. Although there were significant increases in some behavioural determinants from pre- to post-course, pre-course medians for many determinants were already high. As with much health professional assessment, it is hard to know if people are answering as they think or how they believe they should think. The participants were reassured at multiple times of their anonymity and the scores did show variability over time and between participants. Yet, further exploration of the impact of social or professional desirability on the answering of these types of questions is also warranted.

Finally, we found that some questions were not effective for this population, i.e. those around perceived behavioural control and subjective norms. In both of these behavioural determinants, the questions showed low internal consistency, indicating that these were not reliable in measuring an underpinning of the latent variable. We based the development of our questions for these constructs on the REBEQI Manual; therefore, the types of questions had been used before. ${ }^{[15]}$ Measurement of these constructs in Ugandan healthcare professionals would, therefore, require further exploration.

${ }^{*}$ Supporting data. The data supporting the results of this study are available from the corresponding author on request.

Declaration. Consent to participate: all participants were informed that the pre-, post- and follow-up questionnaires were not compulsory to complete; if they chose not to complete them, they were still welcome to fully participate in the training. The MCQ assessment is a standard part of the AIM courses and therefore all participants were expected to complete this.

Acknowledgements. This work would not have been possible without the participation of the clinical officers in Gulu, Uganda. We are very grateful for their time and expertise. The use of AIM was supported by the Greater Manchester Critical Care Skills Institute. The University Hospital of South Manchester Academy and the National Health Service Global Health Exchange supported this work. We are also grateful to the University of Manchester for enabling the involvement of its staff in this study.

Author contributions. Design: LBD, CA, MJ, GJB, JH; data collection: MJJ, RM, HS, AS, SC, SW, HP, SR; data analysis and interpretation: LBD, CA, MJ, JH, JG, GY, HM; write-up: all authors; and final version approval: all authors.

Funding. The data for this research were collected as part of a training course delivered by the Greater Manchester Critical Care Skills Institute in Gulu, Uganda. Travel, accommodation and associated costs were funded by the Greater Manchester Critical Care Skills Institute. Members of the training team collected data, and their time both in delivering the course and collecting data was unfunded, i.e. voluntary (MJJ, RM, HS, AS, SC, SW). Data collection was supported by other self-funded volunteers (HP, SR). Research design, data analysis and write-up were conducted by unfunded volunteers (all authors). There was no formal role for the funding body in the study design, data collection, analysis or write-up. However, authors of the article are active members of the Greater Manchester Critical Care Skills Institute (AS, MJJ, RM, HS, SC, SW). 
Conflicts of interest. AS was the director of the Greater Manchester Critical Care Skills Institute at the time of data collection and write-up. LBD, MJJ, RM, HS, GY, AS, GJB, SC, SW and JH are co-editors of the AIM4Africa course manual, which forms the basis of the training course described in this article.

1. Shelton ID. The six domains of behavior change: The missing health system building block. Glob Heal Sci Pract 2013;1(2):137-140. https://doi.org/10.9745/ghsp-d-13-00083

2. Firth P. Ttendo S. Intensive care in low-income countries - a critical need. N Engl J Med 2012;367:1974-1976. https://doi.org/10.1056/NEJMp1204957

3aker T. Critical care in low-income countries. Trop Med Int Health 2009;14(2):143-148. https://doi.org/10.1111/ j.1365-3156.2008.02202

. McCarthy R, Byrne-Davis L, Hart J, et al. A feasible, acceptable and effective way to teach healthcare workers in low- and middle-income countries: A method to manage acutely ill obstetric women. Midwifery 2015;31(1):19-24 https://doi.org/10.1016/j.midw.2014.04.009

5. Byrne-Davis L, Slattery H, Whiteside S, et al. Efficacy and acceptability of an acute illness management cours delivered to staff and students in Uganda by staff from the UK. Int Health 2014;7(5):360-366. https://doi org/10.1093/inthealth/ihu078

6. Byrne-Davis LM, Byrne GJ, Jackson MJ, et al. Understanding implementation of maternal acute illnes management education by measuring capability, opportunity and motivation: A mixed methods study in a lowincome country. J Nurs Educ Pract 2015;6(3):59. https://doi.org/10.5430/jnep.v6n3p59

7. Michie S, van Stralen MM, West R. The behaviour change wheel: A new method for characterising and designin behaviour change interventions. Implement Sci 2011;6:42. https://doi.org/10.1186/1748-5908-6-42
8. Eccles MP, Grimshaw JM, MacLennan G, et al. Explaining clinical behaviors using multiple theoretical models. Implement Sci 2012;7:99, https://doi.org/10.1186/1748-5908-7-99

9. Presseau J. Johnston M, Heponiemi T, et al. Reflective and automatic processes in health care profession behaviour: A dual process model tested across multiple behaviours. Ann Behav Med 2014;48(3):347-358. https:/ doi.org/10.1007/s12160-014-9609-8

10. Rycroft-Malone J, Seers K, Chandler J, et al. The role of evidence, context, and facilitation in an implementatio trial: Implications for the development of the PARIHS framework. Implement Sci 2013;8:28. https://doi org/10.1186/1748-5908-8-28

11. Bria M, Baban A, Dumitrascu DL. Systematic review of burnout risk factors among European healthcare professionals. Cognitie, Creier, Comportament 2012;16(3):423-452.

12. Zachariah R, Ford N, Philips M, et al. Task shifting in HIV/AIDS: Opportunities, challenges and proposed actions for sub-Saharan Africa. Trans R Soc Trop Med Hyg 2009;103(6):549-558. https://doi.org/10.1016/j. trstmh.2008.09.019

13. Sniehotta FF, Schwarzer R, Scholz U, Schuz B. Action planning and coping planning for long-term lifestyle change: Theory and assessment. Eur J Soc Psychol 2005;35(4):565-576. https://doi.org/10.1002/ejsp.258

14. Sniehotta FF, Scholz U, Schwarzer R. Action plans and coping plans for physical exercise: A longitudinal intervention study in cardiac rehabilitation. Br J Health Psychol 2006;11(1):23-37. https://doi.org/10.1348/ $135910705 \times 43804$

15. Francis AJJ, Eccles MP, Johnston M, et al. Constructive Questionnaires Based on the Theory of Planned Behaviour: A Manual for Health Services Researchers. UK: Centre for Health Services Research, University of Newcastle, 2004:1-42

Accepted 20 June2018. 